\title{
ELS STUDY OF ULTRA THIN AI LAYERS DEPOSITED ON THE Mo(110) FACE
}

\author{
J. KolaczKIEWICZ aNd S. ZUBER \\ Institute of Experimental Plysics, University of Wroclaw \\ Cybulskiego 36, 50-205 Wroclaw, Poland \\ (Received June 6, 1991; in revised form August 27, 1991)
}

\begin{abstract}
Influence of $\mathrm{Al}$ concentration on the energy loss spectrum of the substrate was observed. Also the dependences of the loss amplitude and energy on the primary energy and the layer thickness were studied.
\end{abstract}

PACS numbers: 79.20.-m, 73.20.Mf

\section{Introduction}

Energy loss spectroscopy (ELS) is a source of information on energies of both surface atom excitations and the electron gas collective oscillations - plasmons. According to the theoretical model [1] the plasmon energy depends on the electron gas density. There are two plasma oscillation frequencies $\omega_{\mathrm{b}}$ and $\omega_{\mathrm{s}}$, the latter is attributed to an abrupt change of the electron density at a surface (surface plasmon), while the former is a frequency of the plasma oscillations in the bulk (volume plasmon). Occurrence of the surface and volume plasmons is connected with a free electron gas of density characteristic of a given metal. A metal deposited on a surface of another metal changes the frequency of the substrate plasmons which is a result of mixing of the electron gas from the substrate and adsorbate. When a density of an electron gas of the adsorbate layer reaches a value attributed to a solid also the plasmons with energy characteristic of the adsorbate should appear [2]. So, for thin layers occurrence of both substrate and adsorbate plasmons is expected as long as the penetration depth of the primary electron beam is larger than the adsorbate thickness. For the lowest coverages $\theta \rightarrow 0$ when the electron gas of the substrate and adsorbate is mixed [2] the adsorbate plasmons should not be expected, however, in some papers [3-7] such features were reported and interpreted as plasmon losses.

Our studies concern the appearance of the plasmon losses of the adsorbate and disappearance of the plasmon losses of the substrate during the adsorbate deposition. We chose the aluminium coverage to our study for its relatively simple electronic structure, for this metal the free electron model is true without any exceptions and the excitation energy of the electrons from inner shells is $78 \mathrm{eV}$ or 
more. For $\mathrm{Al}$ the plasmons, which are due to the penetration or reflection of high energy electron beam [8] as well as to the emission of photoelectrons from inner levels [9] or Auger electrons [10], are well known. The earlier work [11] reports a layer-by-layer aluminium growth on the $\mathrm{Mo}(110)$ surface and for low coverage $\left(\theta<1 \mathrm{ML}, 1 \mathrm{ML}=14.12 \times 10^{14} \mathrm{~atm} / \mathrm{cm}^{2}\right)$ annealing at $900 \mathrm{~K}$ changes the surface density of the adsorbate. For these reasons it seems to be an ideal system for our study.

\section{Experimental}

The measurements were performed in UHV system at a pressure of residual gasses of $3 \times 10^{-10}$ torr. The sample surface was perpendicular to the [110] direction with an accuracy of $\pm 0.05^{\circ}$. The surface was thoroughly cleaned by prolongated heating to $1200 \mathrm{~K}$ with simultaneous oxygen exposure (at a pressure of $10^{-7}$ torr) and then subjected to short oxygen exposures and flash-heating at $2000 \mathrm{~K}$. The carbon and molybdenum Auger signal ratio was 1:600, the same was in the case of oxygen and molybdenum. Aluminium was evaporated from a tungsten cup, this source needed a very careful work (especially outgassing of interior parts of the system) to avoid the aluminium oxidation [11].

The energy loss spectra were measured using a spherical analyser with a retarding field. Due to such an analyser the crystallographic changes of the adsorbate layer did not influence the amplitude of the measured losses, the spectra were recorded in $\mathrm{d} N / \mathrm{d} E$ mode, the modulation voltage was $0.5 \mathrm{~V}$ peak-to-peak and the time constant was $1 \mathrm{~s}$. The energy of the electron beam used to investigate the coverage influence on the observed losses was $E_{\mathrm{p}}=94 \mathrm{eV}$. The primary electron current was ranging from a few tenths to a few $\mu \mathrm{A}$, which was dependent on $E_{\mathrm{p}}$.

Measurements of the coverage dependent features were performed on freshly evaporated layers, their thickness was determined using the AES. Studies of the primary energy $E_{\mathrm{p}}$ influence were carried for a few layers of various thicknes for increasing and decreasing $E_{\mathrm{p}}$.values to exclude an influence of residual gasses on the examined surfaces. Each measurement cycle was followed by the measurement of the oxygen and carbon Auger signals, their relation to pure molybdenum did not exceed 1:100.

\section{Results}

The loss spectrum of a thick aluminium layer (thickness exceeding $50 \mathrm{ML}$ was determined from the evaporation time) is shown in Fig. 1. In the Al layer two losses as well as their multiplicities occur. The loss of energy of about $11 \mathrm{eV}$ is visible from the lowest coverage; its amplitude vs. coverage plot is presented in Fig. 2. This amplitude is also influenced by the crystallographic structure of the layer. Changes of the layer structure due to annealing are also shown in Fig. 2. The amplitude and energy changes of the loss attributed to the surface and volume plasmons of the substrate due to the aluminium deposition are presented in Fig. 3. Also the changes of the loss energy and amplitude related to the volume plasmon of the adsorbate are presented in this figure. It follows from the figure that the 


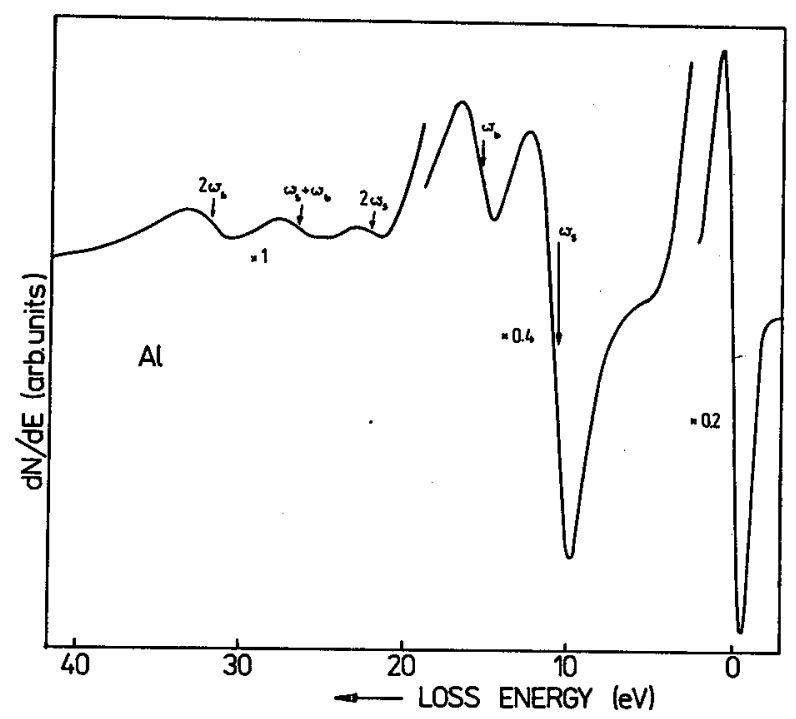

Fig. 1. Characteristic loss spectrum for thick Al layer on the Mo(110) plane, $E_{\mathrm{p}}=$ $94 \mathrm{eV}$.

plasmon amplitude decreases quickly, and gradual changes of the plasmon energy are observed, while the volume plasmon in $\mathrm{Al}$ appears for the $3 \mathrm{ML}$ coverage, but the energy characteristic of this loss is attributed to the loss for the coverage exceeding $10 \mathrm{ML}$.

Dependences of amplitudes and energies of chosen losses on the primary energy are presented in the next three figures. Figures 4 and 5 show the results obtained for two predominant losses in molybdenum and aluminium, respectively. Measurements performed for $0.87 \mathrm{ML}$ aluminium coverage are presented in Fig. 6. This figure shows data obtained for both the adsorbate and substrate. They reveal that the low energy losses (at about $11 \mathrm{eV}$ ) have a maximum at $E_{\mathrm{p}}=200 \mathrm{eV}$. Energy of these losses depend on the primary energy, for $E_{\mathrm{p}}>200 \mathrm{eV}$, the loss energies are increased of about $0.5 \mathrm{eV}$. The results obtained for different coverages agree well with those presented in Fig. 6. The loss amplitudes shown in Figs. 3-6 were normalized to the elastic peak amplitude. The energy was measured between the bending points of the loss and elastic peak (the midpoints of the derivative amplitudes).

\section{Discussion}

The energies of the observed Al losses 11.12 and $15.5 \mathrm{eV}$ are very close to the energies of the surface and volume plasmons in aluminium [8]. In molybdenum the loss energies 22 and $25 \mathrm{eV}$, (the electron gas density is the same in both elements) are considered analogously to tungsten as losses related to the surface and volume plasmons, respectively. The half-width of these losses are large, therefore they 

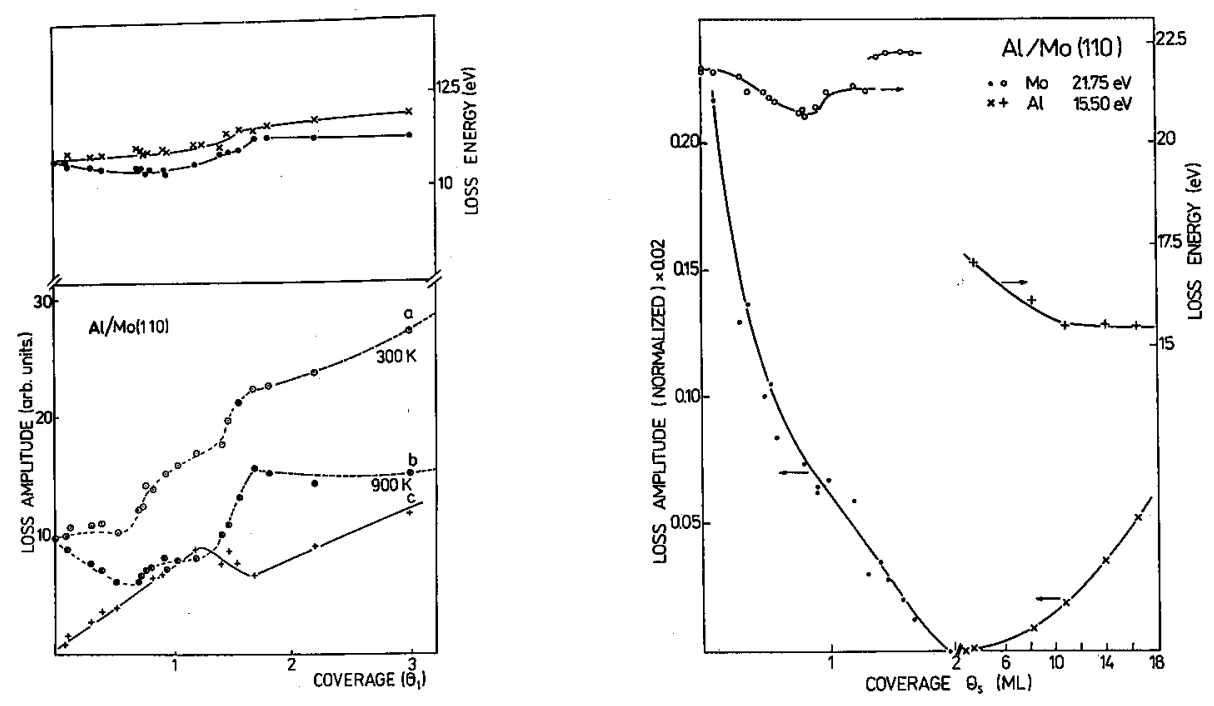

Fig. 2. Change of amplitude (bottom) and energy (top) of loss $\omega_{b}$ vs. coverage. Curve $a$ corresponds to tempeature of $300 \mathrm{~K}$ whereas $b$ of $900 \mathrm{~K}$, respectively. Subtraction $b$ from $a$ results in two straight lines with different slopes, $E_{\mathrm{p}}=94 \mathrm{eV}$

Fig. 3. Change of amplitude (bottom) and energy (top) of losses $\left(\omega_{s}+\omega_{b}\right)$ of Mo and $\omega_{\mathrm{b}}$ of Al vs. coverage.
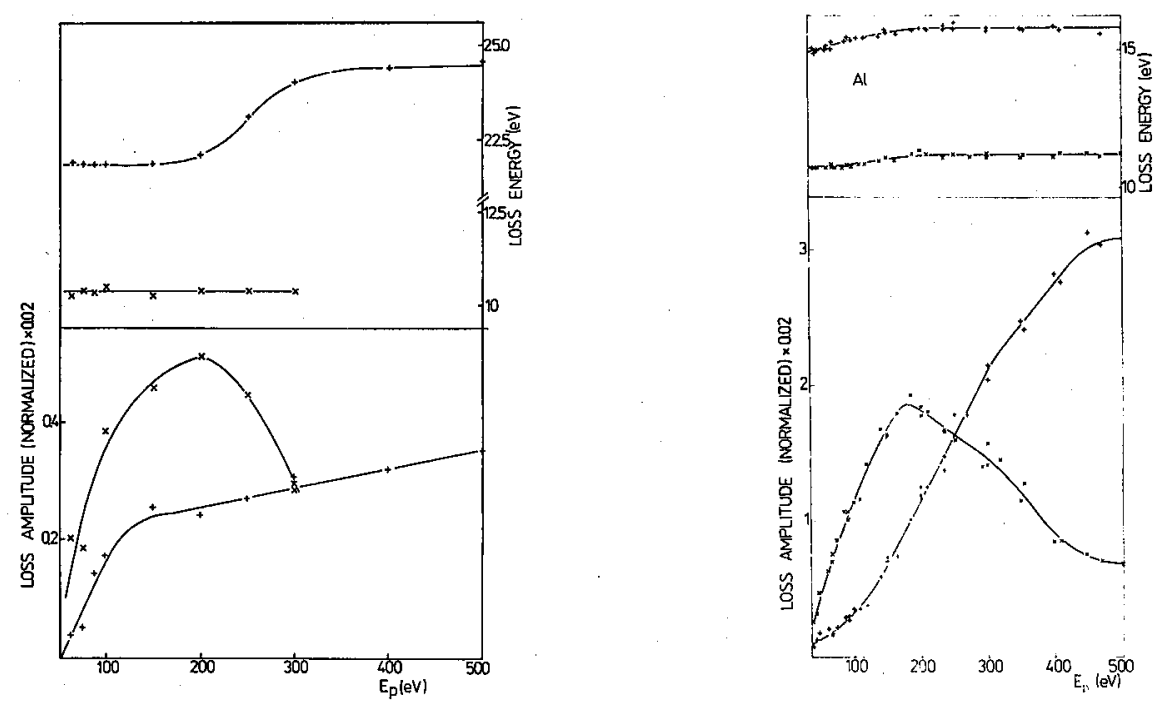

Fig. 4. Amplitude (bottom) and energy (top) of losses with energy of $\Delta E=10.6 \mathrm{eV}$ and $\Delta E=21-24 \mathrm{eV}$ for clean Mo vs. $E_{\mathrm{p}}$

Fig. 5. Amplitude (bottom) and energy (top) of losses $\omega_{b}$ and $\omega_{s}$ vs. primary beam energy for thick (50 ML) Al layers. 


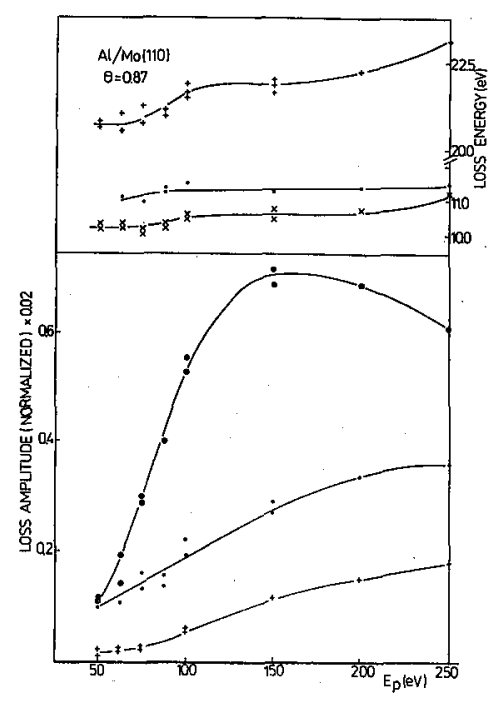

Fig. 6. Amplitude (bottom) and energy (top) of losses observed in the case of Al adlayer $\theta=0.87 \mathrm{ML}$.

cannot be distinguished. Figures 3,4 and 6 show jointly amplitudes and energies of both those features. Ilowever, the interpretation of the $10.8 \mathrm{eV}$ loss in the paper [12] is not clear, the corresponding loss in tungsten is determined as a interband transition. This loss cannot be observed during the $\mathrm{Al}$ evaporation as in $\mathrm{Al}$ there is a loss with similar energy. The coverage dependences of loss amplitudes and energies measured during the aluminium deposition are plotted in Figs. 2 and 3. Considering the fact that the thickness of a layer in which the plasmons can be created by the electron beam of energy of $94 \mathrm{eV}$, cannot exceed $3 \mathrm{ML}$ (the creation depth is 1.5 times larger than the penetration depth [9]) it is not clear why the substrate plasmon disappears when the adsorbate thickness exceeds $2 \mathrm{ML}$. The loss energy decrease agrees with the expected value only for the coverage $\theta \approx 1 \mathrm{ML}$ and then is enhanced non-monotonically to $21.2 \mathrm{eV}$ and for $\theta=1.3 \mathrm{ML}$ abruptly increases to $22.4 \mathrm{eV}$. This coverage is characteristic of not annealed aluminium layer, namely for such coverage a rare $(3 \times 1)$ structure of the layer is transformed into a close $(2 \times 2)$ structure. In the former case the layer density is 0.67 of the substrate density so (1.3 ML corresponds to 2 layers of density 0.67 ), and in the latter case a density is equal to 1.02 of the substrate density. Assuming the surface layer contribution to the electron density of a layer in which the plasmon is created, the energy change should be equal to $2 \mathrm{eV}$, however the observed change is $1 \mathrm{eV}$. On the other hand, energy of the just appearing volume plasmon is of $1.5 \mathrm{eV}$ higher than the value characteristic of a thin layer, which may suggest that the electron density in such a layer is higher than in metallic aluminium due to the contribution of the substrate, but this is inconsistent with theoretical results which indicate that for coverage lower than $1 \mathrm{ML}$ both the substrate and adsorbate plasmons should 
occur [2]. However, the thickness of this layer seems to be too large. We obtained here a discrepancy, for substrate the thickness of the layer in which plasmons may be created is $2 \mathrm{ML}$, while for the adsorbate this thickness is $10 \mathrm{ML}$.

The results presented in Figs. 4-6 suggest that the low-energy losses in both $\mathrm{Mo}$ and $\mathrm{Al}$ are surface dependent. The primary energy increase enlarges the penetration depth and reduces time of the electron transfer through the surface layer, which produces a decrease of the process yield. It can be assumed that the process of generation of these losses is related to the layer which is penetrated by the electrons of energy $200 \mathrm{eV}$. Relations between the amplitudes of the surface and volume plasmons in $\mathrm{Al}$, obtained for high primary energies are consistent with the results obtained for photoelectrons [9]. The loss energy changes related to the primary energy changes which are visible for pure molybdenum can be explained by the fact that for low primary energies the surface plasmon with energy $21 \mathrm{eV}$ predominates, while for $E_{\mathrm{p}}>300 \mathrm{eV}$ the volume plasmon prevails. This may be due to larger penetration depth, which results in changes in relation between both losses, the loss with higher energy increases.

The results obtained for $0.87 \mathrm{ML}$ of $\mathrm{Al}$ coverage are presented in Fig. 6. They show that the surface layer density is different, so the energy of the surface-volume plasmon is lower than for pure Mo, but with increasing primary energy the plasmon energy increases up to the value characteristic of pure Mo. The $10.8 \mathrm{eV}$ loss amplitude increases with decreasing primary energy and reaches a maximum at $E_{\mathrm{p}}=200 \mathrm{eV}$. The surface plasmon of such thin Al layer has a maximum at $E_{\mathrm{p}}=150 \mathrm{eV}$. For the layers of thickness of 1.5 and $0.34 \mathrm{ML}$ these maxima occur at 200 and $100 \mathrm{eV}$, respectively. Similarly, for the annealed layer the maximum occurs at $250 \mathrm{eV}$. After heating the plasmon energy is higher of $0.5 \mathrm{eV}$ due to the change of the layer thickness, the density of the annealed layer is 1.3 of that before annealing, which should change the plasmon energy of $1.5 \mathrm{eV}$. For the annealed layer the maximum occurs at $250 \mathrm{eV}$, the same as a convexity on the curve attributed to the surface plasmon in a very thick aluminium layer. Therefore the observed Al losses may be interpreted as results of two different processes with close energies. The one attributed to lower energy predominates for small coverages and is related to the surface. After annealing the second process of energy higher of about $0.5 \mathrm{eV}$ predominates. This process prevails also for $E_{\mathrm{p}}>250 \mathrm{eV}$. The corresponding loss is related to the layer consisting of close-packed islands, in which the conditions on the plasmon generation are fulfilled. It can be assumed that the electron density in the island is similar to the electron density for $1 \mathrm{ML}$ adsorbate coverage, so this loss may be accumulated with the surface plasmon creation. The loss with lower energy is attributed to the process analogous to the process corresponding to the $10.8 \mathrm{eV}$ loss in Mo. This is indicated by similar dependences of both amplitudes on $E_{\mathrm{p}}$. The annealing of thin layers confines partly this process, the most intensively for $\theta<1.3 \mathrm{ML}$. A physical interpretation of this loss is not clear, however it should not be attributed to the surface plasmon creation because it occurs also for $\theta \rightarrow 0$, when the electron gas of the substrate and adsorbate is mixed [2]. This may be explained as due to excitation of the negative charge connected with the adsorbed atom, or due to the ionization process, however the loss energy is twice as large as the ionization energy of a free atom. So the transfer of all valence electrons into 
the adsorbate should be expected. When a close-packed island is formed (due to annealing) the probability of electron transfer is lower.

\section{Summary}

Despite of relatively simple electronic structure of aluminium and well-known process of plasmon excitation, the interpretation of the results obtained is difficult and complex. It seems that two independent processes with close energies are related to two different mechanisms of the electron excitation.

Another conclusion drawn from the observations is that for the coverage lower than $1 \mathrm{ML}$ the substrate and adsorbate electron gas is mixed. The volume plasmon appears for the coverage exceeding $3 \mathrm{ML}$ and its energy is higher than that for the solid material.

\section{Acknowledgements}

This work was supported by Ministry of National Education.

\section{References}

[1] Ch. Kittel, Introduction to Solid State Physices, PWN, Warszawa 1970, p. 239 (in Polish).

[2] K.F. Wojciechowski, private communicalions.

[3] J. Onsgaard, Ib. Chorkendorff, Phys. Rev. B 33, 3503 (1986).

[4] A. Stenborg, E. Bauer, Phys. Rev. B 36, 5840 (1987).

[5] A. Hohlfeld, K. Horn, Surf. Sci. 211/212, 844 (1989).

[6] F.P. Netzer, J.A.J. Mattlew, in: Handbook on the Physics and Chemistry of Rare Earths, Eds. K.A. Gsclneidner Jr., L. Eyring, S. Hüfner, Vol. 10, North-Holland, Amsterdam 1987, p. 547.

[7] J. Vahakangas, H. Iwasaki, E.D. Williams, R.L. Park, Surf. Sci. 148, 453 (1984).

[8] H. Reather, Excitation of Plasmons and Interband Transitions by Electrons, Springer Tracts in Modern Physics, Eds. G. Höhler, E.A. Niekisch Vol. 88, Springer-Verlag, Berlin 1980.

[9] J. Osterwalder, T. Greber, S. Hüfner, L. Schlapbach, Phys. Rev. B 41, 12495 (1990).

[10] D.L. Seymour, C.F. McConville, D.P. Woodruff, J.E. Inglesfield, Surf. Sci. 214, 57 (1989).

[11] J. Kołaczkiewicz, M. Hochół, S. Zuber, Surf. Sci. 247, 284 (1991).

[12] P.E. Luscher, Surf. Sci. 66, 167 (1977). 